\title{
Changes in the Physical and Mechanical Properties of Human Blood with Sustained Prophylactic Use of Acetylsalicylic Acid (Aspirin) -A Rheological Study
}

\section{Trevor Corrigan, Liam 0'Malley, Dorian Bailey, Hope Moseley, Jada Okaikoi, Thomas Brown, Sean Murray, William Chang, Minseo Yang, Lam Nguyen, Erin Milner, Kevin O'Donovan, Matthew Armstrong}

Department of Chemistry \& Life Science, United States Military Academy, West Point, New York, USA

Email: trevor.corrigan@westpoint.edu

How to cite this paper: Corrigan, $\mathrm{T}$., O'Malley, L., Bailey, D., Moseley, H., Okaikoi, J., Brown, T., Murray, S., Chang, W., Yang, M., Nguyen, L., Milner, E., O’Donovan, K. and Armstrong, M. (2021) Changes in the Physical and Mechanical Properties of Human Blood with Sustained Prophylactic Use of Acetylsalicylic Acid (Aspirin)-A Rheological Study. Open Journal of Fluid Dynamics, 11, 167-176.

https://doi.org/10.4236/ojfd.2021.114010

Received: August 19, 2021

Accepted: November 8, 2021

Published: November 11, 2021

Copyright $\odot 2021$ by author(s) and Scientific Research Publishing Inc. This work is licensed under the Creative Commons Attribution International License (CC BY 4.0).

http://creativecommons.org/licenses/by/4.0/

\begin{abstract}
While the prophylactic use of regular, low dosage, acetylsalicylic acid (aspirin) has been employed due to the inhibitory effect on platelet aggregation, few if any rheological studies validate the change in the physical and mechanical properties of human blood post-aspirin administration. The viscosity and yield stress of blood from eleven donors were assessed at varied shear rates before and after daily aspirin administration $(81 \mathrm{mg})$ for 14 consecutive days. The findings indicate a statistically relevant aggregated decrease with respect to viscosity and yield stress within the study population.
\end{abstract}

\section{Keywords}

Hemorheology, Rouleaux

\section{Introduction}

Aspirin, or acetylsalicylic acid, is one of the most commonly used drugs in the world [1] [2] and is widely utilized for the prophylaxis and treatment of atherosclerotic cardiovascular disease (ASCVD) [2] and arterial thrombotic disorders due to its ability to decrease the viscosity of whole blood and reduce clotting due to platelet aggregation [3] [4]. Recent evidence suggests aspirin may be effective in reducing COVID-19 fatalities associated with thrombosis and pulmonary embolism [5] [6]. While studies have shown that aspirin leads to decreased risk of stroke or other vascular diseases, it is also associated with increased risk of major 
bleeding events [2].

The precursor to aspirin, salicylic acid, was originally isolated from the bark of the willow tree and has been utilized for its anti-pyretic, anti-inflammatory, and analgesic properties for thousands of years [1]. Felix Hoffman synthesized acetylsalicylic acid (aspirin) in 1897 to reduce the gastrointestinal distress associated with carboxylic acid moiety of salicylic acid [7]. Aspirin is rapidly absorbed post oral administration and partially hydrolyzed to salicylate on first pass through the liver [8] [9]. Following oral administration, the maximum concentration (Cmax), attained within the 30 minutes, accumulates in plasma during repeated dosing. The decrease in platelet aggregation is dose dependent [9] and the optimal dose related to the antiplatelet action of aspirin differs per indication relative to the prevention of thrombus formation in the coronary versus vascular circulation.

Aspirin has been shown to inhibit platelet thromboxane production and aggregation, which results in prolonged bleeding times [1]. Mechanistically, aspirin inhibits the synthesis of platelet thromboxane A_2 (TXA2) by irreversibly acetylating a serine reside near the active site of the enzyme cyclooxygenase-1 (COX-1) as shown in Figure 1 [10]. The inhibition of COX-1 results in a reduction in the production of TXA2, which normally stimulates the activation of new platelets, increases platelet aggregation, and functions as a positive-feedback mediator during platelet activation [10] [11]. Since platelets do not synthesize new proteins, the resulting suppression of platelet secretion and aggregation lasts for the life of the platelet (approximately 8 - 12 days) [12]. Platelet aggregation caused by TXA2-independent pathways, such as thrombin formation, remains unchanged during aspirin administration.

While the mechanism and its effect on viscosity are well documented in the literature, few studies have used rheological models and apparatus to explore the

\section{Pre Aspirin Post Aspirin}

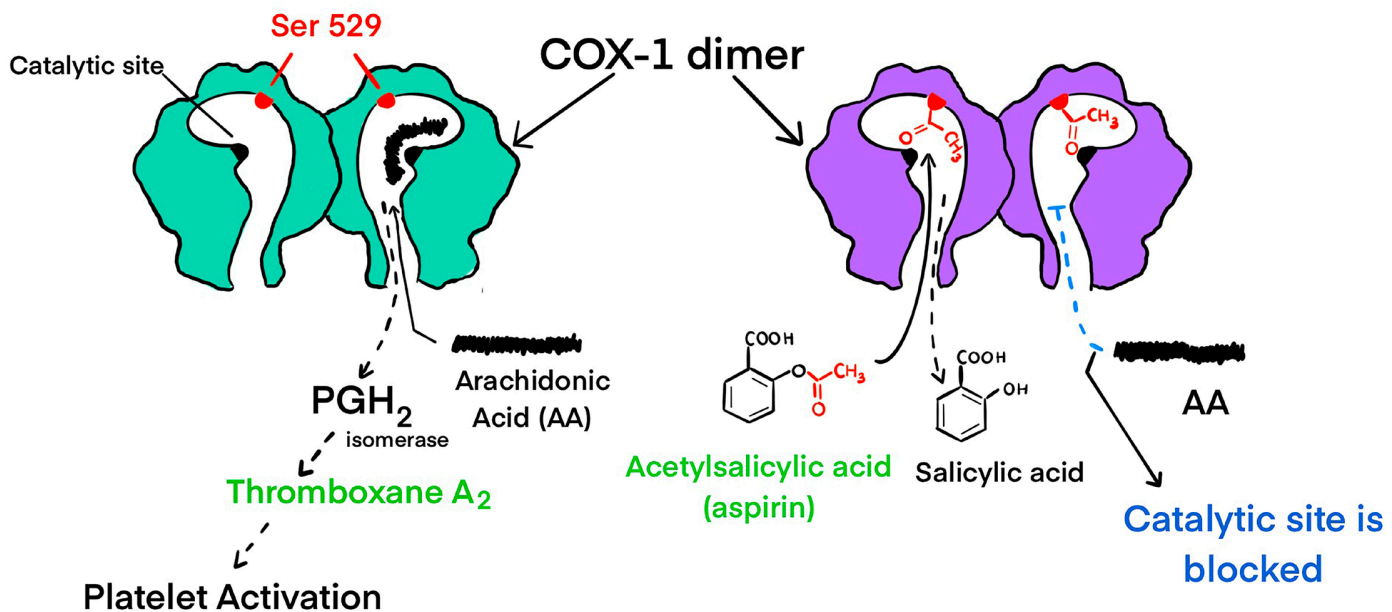

Figure 1. Mechanism of aspirin inhibition of cyclooxygenase-1 catalytic site, preventing the formation of thromboxane A2 and platelet aggregation [10] [11] [13]. Adapted from Fitzgerald \& FitzGerald (2013). 
viscous and elastic properties of blood and the changes in those rheological properties resulting from regular low dosage aspirin use. Hemorheology is the study of the structure, flow and deformation of blood. Human blood is a complex non-Newtonian, fluid fully described as a thixo-elasto-visco-plastic (TEVP) fluid that contains red blood cells, leukocytes, platelets, and various proteins and dissolved solutes [14]-[19]. The thixotropic property of blood means that it has an evolving microstructure that is attributed to rouleaux-the stacking of red blood cells and time-dependent shear thinning properties [14] [18] [19]. The elastic (solid-like: attributed to evolving and stretching rouleaux) and viscous (liquid-like: resistance) properties of blood also allow it to store and dissipate energy (in a nonlinear manner) and undergo deformation and return to its pre-deformed state [15] [17] [18]. The plastic property of blood means that it also has the ability to undergo permanent change in its microstructure after deformation, especially as the blood ages [17] [18]. This TEVP nature of blood is important in order to understand how the flow of blood throughout the human body is variant and dynamic [14]. That is, the physical and mechanical properties of blood and rheological parameters are related to the physiological properties.

For instance, cholesterol and triglyceride content have been shown to affect the rheological parameters of blood [14] [16] [20]. Earlier research related blood viscosity with shear rate, hematocrit levels, and the diameter of the blood vessel in which it flows [14] [21] [22]. There is a direct correlation between the hematocrit level (ratio of the volume of red blood cells to the volume of total blood) and the viscosity of the blood [23] due to the formation of rouleaux structures [24]. Rouleaux are the pseudo-microstructures that red blood cells (RBCs) form at low shear rates that appear as a stack of coins (Figure 2). Fibrinogen is a red blood cell binding protein, known to increase the aggregation of red blood cells into the rouleaux [17]. These factors are known to affect both the yield stress of

\section{Rouleaux Structure}

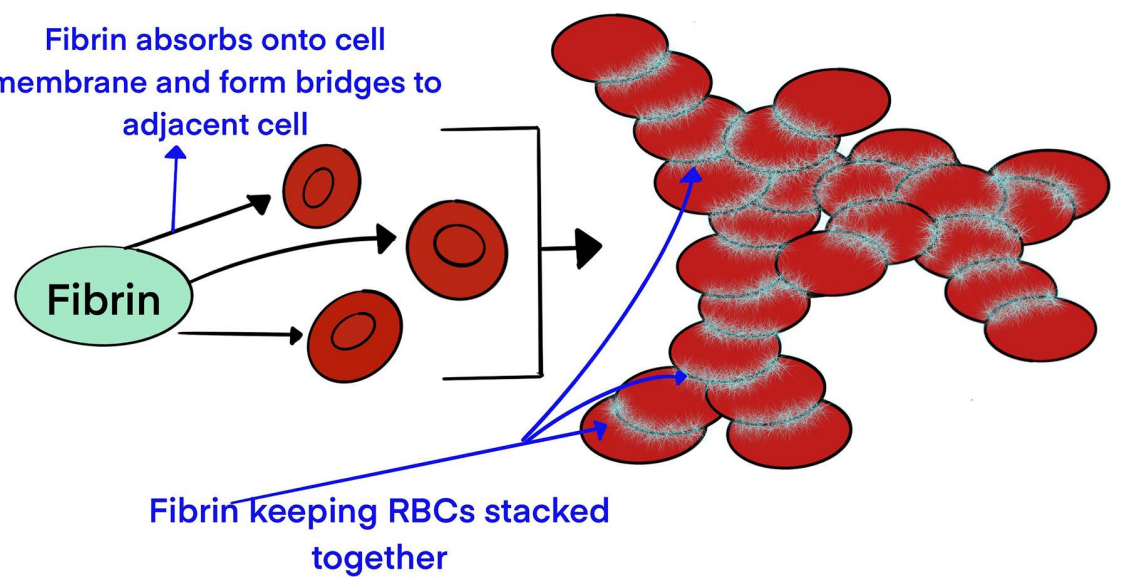

Figure 2. Mechanism by which fibrinogen interacts with red blood cells and forms the rouleaux microstructure, which is one of the major contributors to whole blood viscosity. 
the blood and the absolute viscosity. Studies have shown the value of yield stress varies approximately as the cube of hematocrit and as a function of fibrinogen concentration [25]. The physiological metrics of blood can be used evaluate cardiovascular disease in humans [14] [21] [26] [27] [28]. By modeling the physiological properties, one may predict the incidence of cardiovascular disease by evaluating the various physiological parameters.

The enhanced MHAWB model is a thixo-elasto-visco-plastic model that considers both the thixotropic contributions of the rouleaux formation and the contributions of individual blood cells to shear stress [19]. It combines the WhiteMetzner and Cross models with linear superposition of structural effects. The end result is a model consisting of five ordinary differential equations, six algebraic equations, and ten parameters. Seven of these parameters are used in fitting the steady state rheology data, while three are used when probing the transient data.

The model represents the level of rouleaux formation with the non-dimensional structure parameter, $\lambda$, taking on values [ $\left[\begin{array}{ll}0 & 1\end{array}\right]$, where zero represents blood with all individual RBCs in plasma, and one represents fully "structured" rouleaux. The transient ordinary differential equations that governs rouleaux evolution is a follows

$$
\frac{\mathrm{d} \lambda}{\mathrm{d} t}=\frac{1}{\tau_{\lambda}}\left((1-\lambda)+(1-\lambda) t r_{2}\left|\dot{\gamma}_{p}\right|-t r_{1} \lambda\left|\dot{\gamma}_{p}\right|\right)
$$

where $\tau_{\lambda}, \tau_{a}$, and $\tau_{b}$ are time constants representing the overall rate of structure formation, the relative rate of structure formation due to shearing, and the relative rate of structure breakdown due to shearing, respectively. Under steady shear conditions, the ODE can be simplified to

$$
\lambda_{s s}=\frac{1+t r_{2}|\dot{\gamma}|}{1+t r_{2}|\dot{\gamma}|+t r_{1}|\dot{\gamma}|}
$$

Equation (2) can be used to find the steady state shear rouleaux stress contribution as shown in Equation (3)

$$
\sigma_{S S, R}=\sigma_{y} \lambda_{S S}+\mu_{R} \dot{\gamma}\left(\lambda_{S S}\right)^{1.5}
$$

Additionally, the model represents the individual red blood cell shear stress contributions by a Cross model as seen below in Equation (4)

$$
\sigma_{S S, C}=\left(\frac{\mu_{0, C}-\mu_{\infty, C}}{1+\tau_{C}|\dot{\gamma}|}+\mu_{\infty, C}\right) \dot{\gamma}
$$

where $\tau_{C}$ is a time constant governing the dependence of the apparent viscosity on the shear rate. Finally, to obtain the total stress exhibited by the sample under steady shear conditions, the rouleaux contributions, Equation (3), must be added to the red blood cell contribution, Equation (4),

$$
\sigma_{T o t, S S}=\sigma_{S S, R}+\sigma_{S S, c}
$$

The resulting model has seven parameters, $t r_{2}, t r_{1}, \tau_{c}, \mu_{R}, \mu_{0, C}, \mu_{\infty, C}$ and $\sigma_{y}$, 
which each must be fit to the collected data. To fit the model parameters we have incorporated a stochastic, global optimization algorithm, parallel simulated annealing [29] [30]. Under high shear rates, Equation (3) will go to zero, showing the decreasing contributions of rouleaux structure at high shear. Under low shear rates, the rouleaux contributions dominate the red blood cell contribution, eventually approaching the dynamic yield stress, $\sigma_{y}$, as $\dot{\gamma}$ approaches zero.

\section{Methods}

The handling and measurement protocol employed for the blood rheology results in this work follow the previously established guidelines for blood rheology [14] [18] [19] [29] [30] [31] in compliance with United States Military Academy's Institutional Review Board (RHCA19037_918567). No adverse events were reported by the study participants. The specimens collected were subsequently analyzed within a clinical laboratory (Complete Blood Count and Lipid Panel) per published procedures [18]. All rheological measurements were performed using a Discovery Hybrid Rheometer-3 (DHR-3) stress control rheometer from TA Instruments equipped with a double wall couette geometry following previously published steady state and transient protocols [14]. Venipuncture specimens were obtained at day zero (prior to aspirin dosing) and day 14 post $81 \mathrm{mg}$ daily dosage of aspirin. Measurements are taken using the previously mentioned protocols. The steady state rheological experimental protocol was a log spaced in shear rate progression with a pre-shear of $300 \mathrm{~s}^{-1}$ for $30 \mathrm{~s}$ to remove historical artifacts from previous shear rate measurements; all shear rate tested at a temperature of $37^{\circ} \mathrm{C}$; and all steady states experiments were run in strain-controlled mode [14].

\section{Results}

In Figures 1-4, we show the results of the mHAWB model fitting to 11 sets of steady state data, before and after the aspirin protocol. We focus here on the 4 rheological model parameters that capture the mechanical property change with the aspirin protocol, namely: 1) zero-shear viscosity; 2) infinite-shear viscosity; 3) rouleaux viscosity; and 4) yield stress. These four parameters come directly from fitting the steady state data with a global stochastic optimization algorithm to the mHAWB TEVP model. These results are shown via box and whisker plots in Figures 1-4.

\section{Discussion}

The eleven volunteers in this study were administered $81 \mathrm{mg}$ daily low-dose aspirin regimen for a two-week period. Figure 3 shows the consolidated rheological data for all samples before and after the two-week aspirin protocol. To examine the effect of the aspirin on blood rheology and mechanical properties, we constructed a set of box-and-whisker plots for three different viscosity parameters and one yield stress parameter. The plots show the change in zero shear viscosity (Figure 3), infinite shear viscosity (Figure 4), viscosity due to rouleaux (Figure 5), 


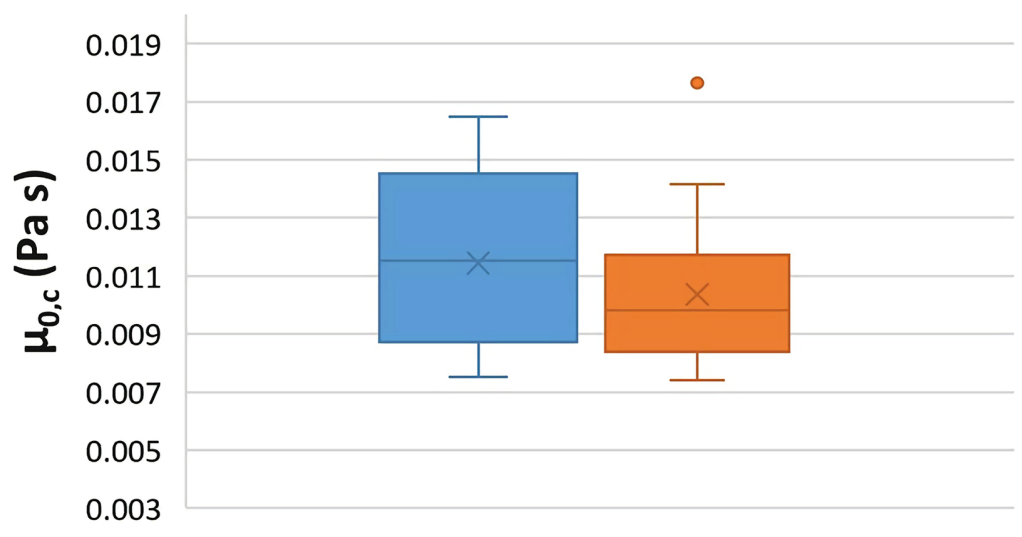

\section{Pre-Aspirin Post-Aspirin}

Figure 3. Box and whisker plot of zero shear viscosity before and after a two-week daily regimen of $81 \mathrm{mg}$ aspirin.

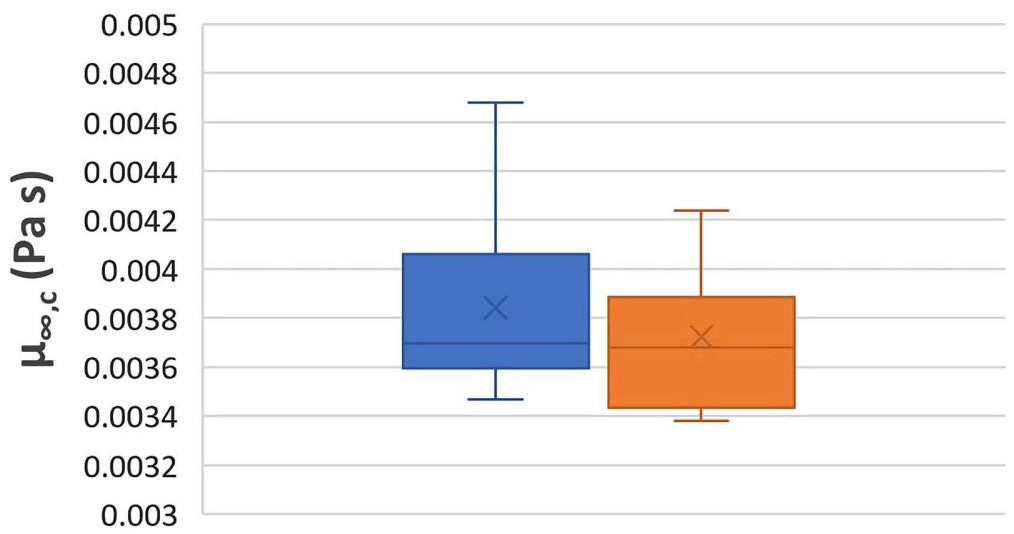

\section{Pre-Aspirin Post-Aspirin}

Figure 4. Box and whisker plot of infinite shear viscosity before and after a two-week daily regimen of $81 \mathrm{mg}$ aspirin.

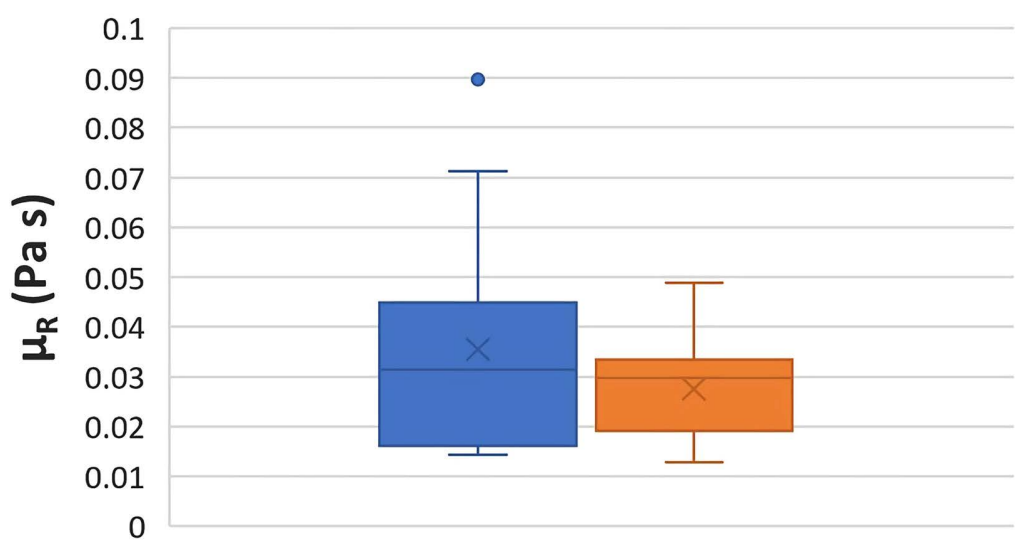

\section{Pre-Aspirin Post-Aspirin}

Figure 5. Box and whisker plot of viscosity due to rouleaux before and after a two-week daily regimen of $81 \mathrm{mg}$ aspirin. 


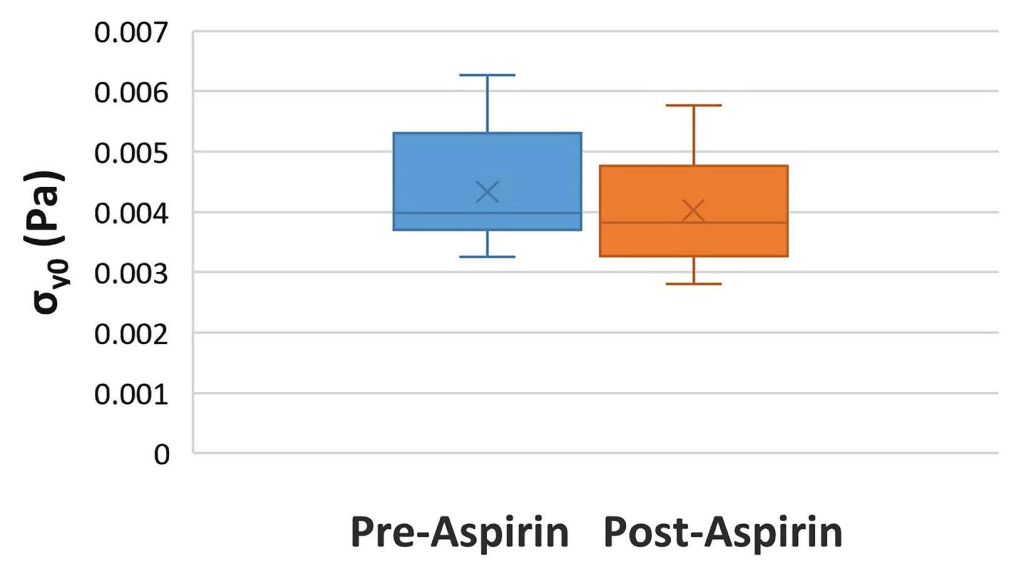

Figure 6. Box and whisker plot of yield stress before and after a two-week daily regimen of $81 \mathrm{mg}$ aspirin

and yield stress (Figure 6) values in the blood of the donors before and after the aspirin administration. While the graphical representations indicate a decrease in blood viscosity post aspirin administration, a paired $\mathrm{t}$-test was performed to determine the significance (if any) of the change in any parameter values. Of the seven parameters, only yield stress and infinite shear viscosity resulted in a p-value less than 0.05 , indicating a significant change.

The data generated during this study utilized a rheometer to probe the complex mechanical properties of human blood more effectively in response to a COX-1 inhibitor (aspirin) which decreases platelet aggregation. Previous studies utilized viscometer, which itself had limited use with low viscosity materials like blood. The DHR-3 rheometer can incorporate the double wall couette geometry increasing surface area, and signal to noise ratio, thereby allowing for an increased shear rate range of experimentation with the human blood. This allowed for interrogation of viscosity at lower and higher shear rates then the viscometer could. The DHR-3 also allowed for more robust experimental control, through rheology experiments conducted at constant stress, or constant shear rate which the viscometer lacks. Thusly, the steady state curve analysis is able to probe viscosities and stresses over four logarithmic decades of shear rates. Although this study is composed of a small sample size, serves as a case study for further analyses regarding the efficacy of hemorheology in probing the more nuanced flow parameters of human blood. The pre and post physiology lab-reporting has shown a relatively constant $( \pm 10 \%)$ on average deviation in the hematocrit, total cholesterol and fibrinogen levels, know to affect the mechanical properties the most, corroborating that the blood viscosity and yield stress was affected in a statistically meaningful way by the one a day $81 \mathrm{mg}$ aspiring protocol for two weeks [18] [19].

\section{Conclusion}

We have demonstrated that regular low dosage aspirin does reduce not just total viscosity but several contributing rheological parameters, such as yield stress. 
Previous studies failed to explore the effects of regular low dosage aspirin on the multiple rheological parameters of healthy adults. The results of this study will serve to fuel further expanded studies into the diagnostic potential of the field of hemorheology. The once a day, $81 \mathrm{mg}$ aspirin protocol for two weeks has shown to induce a statistically significant decrease in viscosity and yield stress of human blood, with all other physiological parameters (cholesterol, fibrinogen, and HCT) remaining relatively constant $\pm 10 \%$ of starting values, aggregated over all 11 donors, from lab pre and post lab reports [32].

\section{Acknowledgements}

The authors acknowledge the support and funding assistance from the U.S. Army, and the Department of Chemistry and Life Science, United States Military Academy. The views expressed herein are those of the authors and do not reflect the position of the United States Military Academy, the Department of the Army, or the Department of Defense.

\section{Data Availability}

The data that support the findings of this study are openly available in Mendeley at Mendeley.com at: DOI: 10.17632/dttytm73pn.1.

\section{Conflicts of Interest}

The authors declare no conflicts of interest regarding the publication of this paper.

\section{References}

[1] Montinari, M.R., Minelli, S. and De Caterina, R. (2019) The First 3500 Years of Aspirin History from Its Roots-A Concise Summary. Vascular Pharmacology, 113, 1-8. https://doi.org/10.1016/j.vph.2018.10.008

[2] Thobani, A., Dhindsa, D.S., DeMoss, B.D., Raad, M., Sandesara, P.B., Sperling, L.S. and Baer, J.T. (2019) Usefulness of Aspirin for Primary Prevention of Atherosclerotic Cardiovascular Disease. American Journal of Cardiology, 124, 1785-1789. https://doi.org/10.1016/j.amjcard.2019.08.040

[3] Verheugt, F.W.A., ten Berg, J.M., Storey, R.F., Cuisset, T. and Granger, C.B. (2019) Antithrombotics: From Aspirin to DOACs in Coronary Artery Disease and Atrial Fibrillation (Part 3/5). Journal of the American College of Cardiology, 74, 699-711. https://doi.org/10.1016/j.jacc.2019.02.080

[4] Patrono, C. (1998) Prevention of Myocardial Infarction and Stroke by Aspirin: Different Mechanisms? Different Dosage? Thrombosis Research, 92, S7-S12. https://doi.org/10.1016/S0049-3848(98)00101-7

[5] Mekaj, A., Mekaj, Y. and Daci, F. (2015) New Insights into the Mechanisms of Action of Aspirin and Its Use in the Prevention and Treatment of Arterial and Venous Thromboembolism. Therapeutics and Clinical Risk Management, 11, 1449-1456. https://doi.org/10.2147/TCRM.S92222

[6] Haque, S., Jawed, A., Akhter, N., Dar, S.A., Khan, F., Mandal, R.K., Areeshi, M.Y., Lohani, M. and Wahid, M. (2020) Acetylsalicylic Acid (Aspirin): A Potent Medicine 
for Preventing COVID-19 Deaths Caused by Thrombosis and Pulmonary Embolism. European Review for Medical and Pharmacological Sciences, 24, 9244-9245.

[7] Mahdi, J.G. (2010) Medicinal Potential of Willow: A Chemical Perspective of Aspirin Discovery. Journal of Saudi Chemical Society, 14, 317-322.

https://doi.org/10.1016/j.jscs.2010.04.010

[8] Levy, G. (1980) Clinical Pharmacokinetics of Salicylates: A Re-Assessment. British Journal of Clinical Pharmacology, 10, 285S-290S. https://doi.org/10.1111/j.1365-2125.1980.tb01811.x

[9] Nagelschmitz, J., Blunck, M., Kraetzschmar, J., Ludwig, M., Wensing, G. and Hohlfeld (2014) Pharmacokinetics and Pharmacodynamics of Acetylsalicylic Acid after Intravenous and Oral Administration to Healthy Volunteers. Clinical Pharmacology: Advances and Applications, 6, 51-59. https://doi.org/10.2147/CPAA.S47895

[10] Undas, A., Brummel-Ziedins, K.E. and Mann, K.G. (2007) Antithrombotic Properties of Aspirin and Resistance to Aspirin: Beyond Strictly Antiplatelet Actions. Blood, 109, 2285-2292. https://doi.org/10.1182/blood-2006-01-010645

[11] Rucker, D.A. (2020) Physiology, Thromboxane A2. StatPearls, Treasure Island.

[12] Davey, R.J. and AuBuchon, J.P. (2007) Chapter 33: Post-Transfusion Red Blood Cell and Platelet Survival and Kinetics: Basic Principles and Practical Aspects. In: Hillyer, C.D., Silberstein, L.E., Ness, P.M., Anderson, K.C., Roback, J.D. and Roback, T.M., Eds., Blood Banking and Transfusion Medicine, Second Edition, Churchill Livingstone, London, 455-466. https://doi.org/10.1016/B978-0-443-06981-9.50038-7

[13] Fitzgerald, D.J. and Fitzgerald, G.A. (2013) Historical Lessons in Translational Medicine: Cyclooxygenase Inhibition and P2Y12 Antagonism. Circulation Research, 112, 174-194. https://doi.org/10.1161/CIRCRESAHA.111.300271

[14] Armstrong, M., Baker, J., Trump, J., Milner, E., Wickiser, J.K., Cameron, K., Clark, N., Schwarting, K., Brown, T., Bailey, D., James, C., Nguyen, C. and Corrigan, T. (2021) Structure-Rheology Elucidation of Human Blood via SPP Framework and TEVP Modeling. Korea-Australia Rheology Journal, 33, 45-63.

https://doi.org/10.1007/s13367-021-0005-1

[15] Apostolidis, A.J. and Beris A.N. (2014) Modeling of the Blood Rheology in SteadyState Shear Flows. Journal of Rheology, 58, 607-633.

https://doi.org/10.1122/1.4866296

[16] Apostolidis, A.J. and Beris, A.N. (2016) The Effect of Cholesterol and Triglycerides on the Steady State Shear Rheology of Blood. Rheologica Acta, 55, 497-509. https://doi.org/10.1007/s00397-015-0889-0

[17] Armstrong, M., Horner, J., Clark, M., Deegan, M., Hill, T., Keith, C. and Mooradian, L. (2018) Evaluating Rheological Models for Human Blood Using Steady State, Transient, and Oscillatory Shear Predictions. Rheologica Acta, 57, 705-728. https://doi.org/10.1007/s00397-018-1109-5

[18] Horner, J.S., Armstrong, M.J., Wagner, N.J. and Beris, A.N. (2019) Measurements of Human Blood Viscoelasticity and Thixotropy under Steady and Transient Shear and Constitutive Modeling Thereof. Journal of Rheology, 63, 799-813. https://doi.org/10.1122/1.5108737

[19] Horner, J.S., Armstrong, M.J., Wagner, N.J. and Beris, A.N. (2018) Investigation of Blood Rheology under Steady and Unidirectional Large Amplitude Oscillatory Shear. Journal of Rheology, 62, 577-591. https://doi.org/10.1122/1.5017623

[20] Moreno, L., Calderas, F., Sanchez-Olivares, G. and Medina-Torres, L. (2015) Effect of Cholesterol and Triglycerides Levels on the Rheological Behavior of Human 
Blood. Korea- Australia Rheology Journal, 27, 1-10.

https://doi.org/10.1007/s13367-015-0001-4

[21] Apostolidis, A.J., Armstrong, M.J. and Beris, A.N. (2015) Modeling of Human Blood Rheology in Transient Shear Flows. Journal of Rheology, 59, 275-298.

https://doi.org/10.1122/1.4904423

[22] Reasor, C., Clausen, D. and Aidun, J. (2013) Rheological Characterization of Cellular Blood in Shear. Journal of Fluid Mechanics, 726, 497-516. https://doi.org/10.1017/jfm.2013.229

[23] Çinar, Y., Demir, G., Paç, M. and Çinar, A.B. (1999) Effect of Hematocrit on Blood Pressure via Hyperviscosity. American Journal of Hypertension, 12, 739-743. https://doi.org/10.1016/S0895-7061(99)00011-4

[24] Tomaiuolo, G., Carciati, A., Caserta, S. and Guido, S. (2016) Blood Linear Viscoelasticity by Small Amplitude Oscillatory Flow. Rheologica Acta, 55, 485-495. https://doi.org/10.1007/s00397-015-0894-3

[25] Merrill, E.W. and Pelletier, G.A. (1967) Viscosity of Human Blood: Transition from Newtonian to Non-Newtonian. Journal of Applied Physiology, 23, 178-182. https://doi.org/10.1152/jappl.1967.23.2.178

[26] Banyai, S., Banyai, M., Falger, J., Jansen, M. and Alt, E. (2001) Atorvastatin Improves Blood Rheology in Patients with Familial Hypercholesterolemia (FH) on Long-Term LDL Apheresis Treatment. Atherosclerosis, 159, 513-519. https://doi.org/10.1016/S0021-9150(01)00532-9

[27] Destiana, D. and Timan, I.S. (2018) The Relationship between Hypercholesterolemia as a Risk Factor for Stroke and Blood Viscosity Measured Using Digital Microcapillary. Journal of Physics: Conference Series, 1073, Article ID: 042045. https://doi.org/10.1088/1742-6596/1073/4/042045

[28] Lee, H., Na, W., Lee, S.B., Ahn, C.W., Moon, J.S., Won, K.C. and Shin, S. (2019) Potential Diagnostic Hemorheological Indexes for Chronic Kidney Disease in $\mathrm{Pa}$ tients With Type 2 Diabetes. Frontiers in Physiology, 10, Article No. 1062. https://doi.org/10.3389/fphys.2019.01062

[29] Bureau, M., Healy, J.C., Bourgoin, D. and Joly, M. (1980) Rheological Hysteresis of Blood at Low Shear Rate. Biorheology, 17, 191-203. https://doi.org/10.3233/BIR-1980-171-221

[30] Armstrong, M.J., Beris, A.N. and Wagner, N.J. (2017) An Adaptive Parallel Tempering Method for the Dynamic Data-Driven Parameter Estimation of Nonlinear Models. AIChE Journal, 63, 1937-1958. https://doi.org/10.1002/aic.15577

[31] Bureau, M., Healy, J.C., Bourgoin, D. and Joly, M. (1979) Etude rhéologique en régime transitoire de quelques échantillons de sangs humains artificiellement modifiés. Rheologica Acta, 18, 756-768. https://doi.org/10.1007/BF01533351

[32] Armstrong, M. and Corrigan, T. (2021) USMA Aspirin Study-Pre and Post Human Blood Rheology. Mendeley Data, V1.

https://data.mendeley.com/datasets/dttytm73pn/1 